TRANSACTIONS OF THE

AMERICAN MATHEMATICAL SOCIETY

Volume 349, Number 9, September 1997, Pages 3517-3534

S 0002-9947(97)01823-0

\title{
MONOID HECKE ALGEBRAS
}

\author{
MOHAN S. PUTCHA
}

\begin{abstract}
This paper concerns the monoid Hecke algebras $\mathcal{H}$ introduced by Louis Solomon. We determine explicitly the unities of the orbit algebras associated with the two-sided action of the Weyl group $W$. We use this to:

1. find a description of the irreducible representations of $\mathcal{H}$,

2. find an explicit isomorphism between $\mathcal{H}$ and the monoid algebra of the Renner monoid $R$,

3. extend the Kazhdan-Lusztig involution and basis to $\mathcal{H}$, and

4. prove, for a $W \times W$ orbit of $R$, the existence (conjectured by Renner) of generalized Kazhdan-Lusztig polynomials.
\end{abstract}

\section{INTRODUCTION}

A monoid analogue of the Iwahori-Hecke algebra [11] was obtained by Solomon [27]-[29]. In an earlier paper [19] the author studied Solomon's monoid Hecke algebras by studying the associated orbit algebras. These orbit algebras arise from the two-sided action of the Weyl group $W$ on the Renner monoid $R$. In particular, the coefficients of the unity of the empty level orbit algebra were shown to be $\bar{R}_{x, y}$, where $R_{x, y}$ are polynomials introduced by Kazhdan and Lusztig [12]. The other orbit algebras were also shown to have unities, but their coefficients were only implicitly given. In this paper we give an explicit formula for the unities of all the orbit algebras, thereby obtaining a description of the irreducible representations of monoid Hecke algebras. We also obtain an explicit, but very complicated, isomorphism between the monoid Hecke algebra and the monoid algebra of $R$, solving a problem posed by Solomon [28]. We go on to extend to the monoid Hecke algebra the Kazhdan-Lusztig involution and basis for the (group) Iwahori-Hecke algebra. This then immediately yields polynomials $P_{\theta, \sigma}$ for $\theta, \sigma$ in the same $W \times W$ orbit of $R$, partially solving a problem posed by Renner [26]. These polynomials are still mysterious; however, in the simplest case they are products of relative KazhdanLusztig polynomials introduced by Deodhar [6].

\section{Reductive monoids AND MONOIDS OF Lie Type}

Consider the general linear group $G=G L_{n}(F)$ over an algebraically closed field $F$. It is the unit group of the multiplicative monoid $M=M_{n}(F)$ of all $n \times n$ matrices over $F$. This monoid has the following structure. The diagonal idempotents form a Boolean lattice with respect to the natural order of idempotents:

$$
f \leq e \quad \text { if } \quad \text { ef }=f e=f .
$$

Received by the editors December 3, 1993.

1991 Mathematics Subject Classification. Primary 20G40, 20G05, 20M30.

Research partially supported by NSF Grant DMS9200077.

(c)1997 American Mathematical Society 
The $G \times G$ orbits have a cross-section $\Lambda$ of idempotents

$$
e_{r}=\left[\begin{array}{cc}
I_{r} & 0 \\
0 & 0
\end{array}\right] .
$$

These idempotents and hence the $G \times G$ orbits are linearly ordered according to rank. The orbit $J_{r}=G e_{r} G$ consists of all rank $r$ matrices and gives rise to the orbit semigroup

$$
J_{r}^{0}=G e_{r} G \cup\{0\}
$$

where for $a, b \in J_{r}$,

$$
a \cdot b= \begin{cases}a b & \text { if } a b \in J_{r} \\ 0 & \text { otherwise }\end{cases}
$$

We also have the orbit monoid

$$
M\left(J_{r}\right)=G \cup J_{r}^{0}
$$

The idempotents in $J_{r}$ are all conjugate, and hence $M\left(J_{r}\right)$ is completely determined by the parabolic subgroups

$$
\begin{gathered}
P_{r}=\left\{g \in G \mid g e_{r}=e_{r} g e_{r}\right\}=\left\{\left[\begin{array}{cc}
A & B \\
0 & C
\end{array}\right] \mid A \in G L_{r}\right\}, \\
P_{r}^{-}=\left\{g \in G \mid e_{r} g=e_{r} g e_{r}\right\}=\left\{\left[\begin{array}{ll}
A & 0 \\
B & C
\end{array}\right] \mid A \in G L_{r}\right\},
\end{gathered}
$$

and the natural homomorphisms $\delta_{+}: P_{r} \rightarrow G L_{r}$ and $\delta_{-}: P_{r}^{-} \rightarrow G L_{r}$. We write $M\left(J_{r}\right)$ as $M\left(G, P_{r}, P_{r}^{-}, G L_{r}\right)$ with $\delta_{+}, \delta_{-}$being understood to be part of the data. There are two deficiencies with the monoid $M$. First, only maximal parabolics arise as $P_{r}$, and second, the orbit monoids are not submonoids of $M$. Both of these drawbacks can be handled by considering the representation $\theta$ of $M$ given by

$$
\theta(a)=\wedge^{1}(a) \otimes \wedge^{2}(a) \otimes \cdots \otimes \wedge^{n}(a)
$$

the tensor product of all exterior powers of $a$. The representation $\theta$ has the effect of killing the singular part of $M$, because $\theta(M)=\theta(G) \cup\{0\}$. However, taking the Zariski closure of $\theta(M)$ yields an amazing monoid $\widetilde{M}$. Again there is a $G \times G$ cross-section $\Lambda$ of idempotents, but this time $\Lambda \backslash\{0\}$ is a Boolean lattice isomorphic to the power set of the Dynkin diagram of $G$. Moreover, any parabolic subgroup is associated with a unique orbit monoid, which now is a submonoid of $\widetilde{M}$. Further, the lattice of diagonal idempotents is the dual of the Coxeter complex.

Both $M$ and $\widetilde{M}$ are examples of reductive monoids. The theory of reductive monoids has been well developed by Renner and the author; cf. [17]. In particular the author [16] has proved the existence of a cross-section lattice $\Lambda$ and has shown that the orbit monoids are of the form $M\left(G, P, P^{-}, L / K\right)$ for some pair of opposite parabolics $P, P^{-}, K \triangleleft L=P \cap P^{-}$. Renner [24] has shown the existence of the finite inverse monoid

$$
R=\langle W, \Lambda\rangle,
$$

where $W$ is the Weyl group of $G$ such that

$$
M=\bigsqcup_{\sigma \in R} B \sigma B,
$$

where $B$ is the Borel subgroup $G$. We call $R$ the Renner monoid of $M$. 
The theory of monoids of Lie type is an attempt to accomplish the above for finite groups of Lie type. Let $G$ be a finite group of Lie type with Weyl group $W$ and Borel subgroup $B$. The most convenient definition of a monoid $M$ of Lie type (with unit group $G$ ) is also the most general, and is due to the author [18]. The definition is simply that all the orbit monoids are of the form

$$
M\left(G, P, P^{-}, L / K\right),
$$

where $P, P^{-}$are opposite parabolic subgroups, $L=P \cap P^{-}, K \triangleleft L$. These monoids have been classified by the author [18], [20] using the theory of buildings [30] and the ideas of Renner and the author [21] for reductive monoids. Renner and the author [22] obtained an analogue of the canonical monoid $\widetilde{M}$ for any finite group $G$ of Lie type.

Let $M$ be a monoid of Lie type with unit group $G$. There is again a crosssection lattice $\Lambda$ for the $G \times G$ orbits. There is also an analogue of Renner monoid $R=\langle W, \Lambda\rangle$ such that

$$
M=\bigsqcup_{\sigma \in R} B \sigma B .
$$

We refer to [18] for details. We will let $\leq$ and $\ell$ denote the Bruhat order and the length function, respectively, on the Weyl group $W$; cf. [10]. For $e \in \Lambda$, let

$$
\begin{aligned}
W(e) & =\{x \in W \mid x e=e x\}, \\
W_{e} & =\{x \in W \mid x e=e\} \triangleleft W(e) .
\end{aligned}
$$

Then both $W(e)$ and $W_{e}$ are parabolic subgroups of $W$, and $W(e)$ is the direct product of $W_{e}$ and the Weyl group of the unit group of $e M e$. Let

$$
\begin{aligned}
D(e) & =\{x \in W \mid x \text { is of minimum length in } x W(e)\}, \\
D_{e} & =\left\{x \in W \mid x \text { is of minimum length in } x W_{e}\right\} .
\end{aligned}
$$

Now

$$
R=\bigsqcup_{e \in \Lambda} W e W
$$

and each element $\sigma \in W e W$ can be uniquely written as

$$
\sigma=x e y^{-1}, \quad x \in D_{e}, y \in D(e) .
$$

We call this the standard form of $\sigma$. If $w_{0}, v_{0}$ are respectively the longest elements of $W$ and $W(e)$, then $w_{0} v_{0}$ is the longest element of $D(e)$. Following Solomon [27], [29] and Renner [25], we let

$$
\ell(e)=\ell\left(w_{0} v_{0}\right)
$$

and, for $\sigma=x e y^{-1}$ in standard form,

$$
\ell(\sigma)=\ell(x)+\ell(e)-\ell(y) .
$$

It is shown in [25] that

$$
\ell(\sigma)=0 \text { if and only if } \sigma B=B \sigma .
$$

If $\sigma=x e y^{-1}$ and $\theta=s f t^{-1}$ in standard form, then define

$$
\sigma \leq \theta \quad \text { if } \quad e \leq f \text { and } x \leq s w, t w \leq y \text { for some } w \in W(f) W_{e} .
$$


We have shown in [15] that in the analogous situation for reductive monoids, $\sigma \leq \theta$ if and only if $B \sigma B$ is contained in the Zariski closure of $B \theta B$. For this reason we say that $\sigma$ is triangular if $\sigma \leq 1$. Thus, if $\sigma=x e y^{-1}$ in standard form,

$$
\sigma \text { is triangular if and only if } x \leq y \text {. }
$$

If $\sigma \in W e W$ and $\theta \in W e W$, then

$$
\sigma \leq \theta \quad \text { implies } \quad \ell(\sigma) \leq \ell(\theta) .
$$

\section{HeCke ALGEBRAS}

Let $G$ be a Chevalley group defined over $\mathbb{F}_{q}, B, B^{-}$opposite Borel subgroups of $G, T=B \cap B^{-}, B=U T, B^{-}=U^{-} T$, and $W$ the Weyl group of $G$ with generating set $S$ of simple reflections. If $x \in W$, let $\dot{x}$ denote a coset representative in $G$. Let

$$
\epsilon=\frac{1}{|B|} \sum_{b \in B} b \in \mathbb{C}[G] .
$$

The Iwahori-Hecke algebra

$$
H_{\mathbb{C}}(G)=H_{\mathbb{C}}(G, B)=\epsilon \mathbb{C}[G] \epsilon
$$

is a semisimple algebra that is isomorphic to $\mathbb{C}[W]$ by a theorem of Tits (cf. [1], [4]). Clearly $H_{\mathbb{C}}(G, B)$ has a basis

$$
A_{x}=\epsilon x \epsilon, \quad x \in W
$$

This basis is normalized as

$$
T_{x}=q^{\ell(x)} A_{x}, \quad x \in W .
$$

With respect to this basis, Iwahori [11] showed that the structure constants are integer polynomials in $q$, depending only on $W$. In particular,

$$
T_{x y}=T_{x} T_{y}, \quad A_{x y}=A_{x} A_{y} \quad \text { if } \ell(x y)=\ell(x)+\ell(y) .
$$

One can therefore consider the generic Hecke algebra $\mathcal{H}(W)$ with basis $T_{x}(x \in W)$ over $\mathbb{Z}\left[q^{1 / 2}, q^{-1 / 2}\right]$, where $q$ is now being treated as an indeterminate. Specifying $q$ and tensoring with $\mathbb{C}$, one recovers $H_{\mathbb{C}}(G, B)$. Kazhdan and Lusztig [12] introduced an involution on $\mathcal{H}(W)$ given by

$$
\overline{q^{1 / 2}}=q^{-1 / 2}, \quad \bar{T}_{x}=T_{x^{-1}}^{-1}=\sum_{y \in W} q^{-\ell(y)} \bar{R}_{y, x} T_{y},
$$

where $R_{y, x}=R_{y, x}(q) \in \mathbb{Z}[q]$. These polynomials are nonzero exactly when $y \leq x$, and have been studied in detail by Deodhar [5]. We see by (3) that

$$
\bar{A}_{x}=A_{x^{-1}}^{-1}=q^{\ell(x)} \sum_{y \in W} \bar{R}_{y, x} A_{y} .
$$

Now let $M$ be a monoid of Lie type with unit group $G$, cross-section lattice $\Lambda$ and Renner monoid $R$. We let $\mathbb{C}[M]$ denote the complex monoid algebra of $M$. By a result of Okniński and the author $[14], \mathbb{C}[M]$ is always semisimple. Following Solomon [27]-[29], we introduce the monoid Hecke algebra

$$
H_{\mathbb{C}}(M)=H_{\mathbb{C}}(M, B)=\epsilon \mathbb{C}[M] \epsilon .
$$


Since $\mathbb{C}[M]$ is semisimple, so is $H_{\mathbb{C}}(M, B)$. Using this fact, Solomon [27], [29] has proved that $H_{\mathbb{C}}(M, B) \cong \mathbb{C}[R]$. This algebra has been further studied by the author [19]. Clearly, $H_{\mathbb{C}}(M, B)$ has a basis,

$$
A_{\sigma}=\epsilon \sigma \epsilon, \quad \sigma \in R
$$

Following Solomon [27], [29], we normalize this basis as

$$
T_{\sigma}=q^{\ell(\sigma)} A_{\sigma}, \quad \sigma \in R .
$$

Fix $e \in \Lambda$. Then for some $I \subseteq S$ we have $W(e)=W_{I}$ and

$$
\begin{aligned}
P_{I} & =\{x \in G \mid x e=e x e\}, \\
P_{I}^{-} & =\{x \in G \mid e x=e x e\} .
\end{aligned}
$$

Let $L=P_{I} \cap P_{I}^{-}$. So $P_{I}=L U_{I}$ and $P_{I}^{-}=L U_{I}^{-}$, where $U_{I}$ and $U_{I}^{-}$are the unipotent radicals of $P_{I}$, and $P_{I}^{-}$, respectively. Then $U_{I} e=e U_{I}^{-}=\{e\}$ and $B_{L}=B \cap L$ is a Borel subgroup of $L$. By [1, Propositions 2.3.3 and 2.5.15],

$$
y B_{L} y^{-1} \subseteq B, y^{-1} B y \subseteq U_{I}^{-} B \quad \text { for all } y \in D(e) .
$$

Hence, for all $y \in D(e)$,

$$
A_{e y^{-1}} A_{y}=\epsilon e y^{-1} \epsilon y \epsilon=\epsilon e \epsilon=A_{e} .
$$

So

$$
A_{e y^{-1}}=A_{e} A_{y}^{-1}=A_{e} \bar{A}_{y^{-1}} \quad \text { for all } y \in D(e) .
$$

Let $x \in W$. Then for $b \in B$, be $=e b_{1}$ for some $b_{1} \in B_{L}$. So for $y \in D(e)$,

$$
\begin{aligned}
\epsilon x b e y^{-1} \epsilon & =\epsilon x e b_{1} y^{-1} \epsilon \\
& =\epsilon x e y^{-1} \cdot y b_{1} y^{-1} \cdot \epsilon \\
& =\epsilon x e y^{-1} \epsilon, \quad \text { by }(7) .
\end{aligned}
$$

Thus $A_{x} A_{e y^{-1}}=A_{x e y^{-1}}$. So by (8),

$$
A_{x e y^{-1}}=A_{x} A_{e} \bar{A}_{y^{-1}} \quad \text { for } x \in W, y \in D(e) .
$$

Now let

$$
\epsilon_{1}=\frac{1}{\left|B_{L}\right|} \sum_{b \in B_{L}} b, \quad \epsilon_{2}=\frac{1}{\left|U_{I}\right|} \sum_{u \in U_{I}} u
$$

Then $\epsilon=\epsilon_{1} \epsilon_{2}=\epsilon_{2} \epsilon_{1}$. So for $z \in W(e)$,

$$
\begin{aligned}
& A_{z} A_{e}=\epsilon z \epsilon e \epsilon=\epsilon z e \epsilon=\epsilon e z \epsilon \\
& \quad=\epsilon e z \epsilon_{2} \epsilon=\epsilon e \epsilon_{2} z \epsilon=\epsilon \epsilon_{1} e \epsilon_{2} z \epsilon=\epsilon e \epsilon_{1} \epsilon_{2} z \epsilon \\
& =A_{e} A_{z} .
\end{aligned}
$$

So, by (9),

$$
A_{z e}=A_{z} A_{e}=A_{e} A_{z} \quad \text { for } z \in W(e) .
$$

Now let $e, f \in \Lambda$ and $x \in W$. Then $x=z y^{-1}$ for some $z \in W(e)$ and $y \in D(e)$. So

$$
\begin{aligned}
A_{e} \bar{A}_{x} A_{f} & =A_{e} \bar{A}_{z} \bar{A}_{y^{-1}} A_{f}, \quad \text { by }(3) \\
& =\bar{A}_{z} A_{e} \bar{A}_{y^{-1}} A_{f}, \quad \text { by }(5),(10) \\
& =\bar{A}_{z} A_{e y^{-1}} A_{f}, \quad \text { by }(8) \\
& =\bar{A}_{z} A_{e y^{-1} f} .
\end{aligned}
$$


So by (5), (9), (10), we have

Theorem 2.1. The structure constants of $H_{\mathbb{C}}(M, B)$ with respect to the basis $A_{\sigma}$ $(\sigma \in W)$ and hence with respect to the basis $T_{\sigma}(\sigma \in w)$ are integer Laurent polynomials in $q$, depending only on $R$.

Remark 2.2. Solomon [27], [29] has actually proved much more. He showed that $H_{\mathbb{C}}(M, B)$ has a presentation given by

$$
\begin{aligned}
& T_{s} T_{\sigma}= \begin{cases}q T_{\sigma} & \text { if } \ell(s \sigma)=\ell(\sigma), \\
T_{s \sigma} & \text { if } \ell(s \sigma)=\ell(\sigma)+1, \\
q T_{s \sigma}+(q-1) T_{\sigma} & \text { if } \ell(s \sigma)=\ell(\sigma)-1,\end{cases} \\
& T_{\nu} T_{\sigma}=q^{\ell(\sigma)-\ell(\nu \sigma)} T_{\nu \sigma}
\end{aligned}
$$

for $s \in S, \sigma, \nu \in R, \ell(\nu)=0$.

Because of Theorem 2.1 we can consider the generic monoid Hecke algebra $\mathcal{H}(R)$ with basis $A_{\sigma}(\sigma \in R)$ or equivalently $T_{\sigma}(\sigma \in R)$ over $\mathbb{Z}\left[q^{1 / 2}, q^{-1 / 2}\right]$, where $q$ is now being treated as an indeterminate. Specifying $q$ and tensoring with $\mathbb{C}$, one recovers $H_{\mathbb{C}}(M, B)$.

\section{Orbit Algebras}

The idea here is very simple and is a key ingredient of semigroup representation theory (cf. [2, Chapter 5]): If $\mathcal{I}$ is an ideal of a semisimple algebra $\mathcal{A}$, then $\mathcal{A} \cong$ $\mathcal{I} \oplus \mathcal{A} / \mathcal{I}$. Let $M$ be a monoid of Lie type with unit group $G$, cross-section lattice $\Lambda$, Weyl group $W$ and Renner monoid $R$. Since $\mathbb{C}[M]$ is semisimple by [14], we have

$$
\mathbb{C}[M] \cong \bigoplus_{J} \mathbb{C}_{0}\left[J^{0}\right]
$$

where the summation is over the $G \times G$ orbits $J$ and $\mathbb{C}_{0}\left[J^{0}\right]$ is the contracted semigroup algebra; i.e. the zero of $J^{0}$ is the zero of the algebra. Making the isomorphism in (11) explicit is a difficult open problem. For a $G \times G$ orbit $J$, let

$$
H_{\mathbb{C}}(J)=H_{\mathbb{C}}(J, B)=\epsilon \mathbb{C}_{0}\left[J^{0}\right] \epsilon .
$$

Call this an orbit Hecke algebra. As in (11), if we use the natural order on $\Lambda$ and proceed inductively, beginning with the least element of $\Lambda$, we have

$$
H_{\mathbb{C}}(M, B) \cong \bigoplus_{J} H_{\mathbb{C}}(J, B),
$$

where the summation is over the $G \times G$ orbits $J$. We will make the isomorphism in (12) explicit in Theorem 3.3. We note that $H_{\mathbb{C}}(J, B)$ is an ideal of $H_{\mathbb{C}}(M, B) / \mathcal{I}$, where

$$
\mathcal{I}=\sum_{J^{\prime}<J} H_{\mathbb{C}}\left(J^{\prime}, B\right) .
$$

Hence

$$
\widehat{H}_{\mathbb{C}}(J)=\widehat{H}_{\mathbb{C}}(J, B)=H_{\mathbb{C}}(J, B)+H_{\mathbb{C}}(G, B)
$$

is a subalgebra of $H_{\mathbb{C}}(M, B) / \mathcal{I}$. We call it the augmented orbit Hecke algebra. Now fix $e \in \Lambda$ and let $J=G e G$. Let $I \subseteq S, P_{I}, P_{I}^{-}, L, B_{L}$ be as in (6). The author [19, Section 2] has shown that if $x, y \in D(e)$, then, in $H_{\mathbb{C}}(J)$,

$$
A_{e x^{-1}} A_{y e}=Q_{x, y} A_{e},
$$


where

$$
Q_{x, y}=\sum_{w \in W(e)} \frac{\left|x^{-1} B y \cap U_{I}^{-} B_{L} w B\right|}{|B|} A_{w} .
$$

Let $P=P_{I}$ and $P^{-}=P_{I}^{-}$, and consider the natural map

$$
\xi: P^{-} P=U_{I}^{-} L U_{I} \rightarrow L
$$

If $w \in W(e)$, then

$$
\xi\left(B^{-} w B\right)=B_{L}^{-} w B_{L}=\bigsqcup_{z \in W(e)}\left(B_{L}^{-} w B_{L} \cap B_{L} z B_{L}\right) .
$$

Let $x, y \in D(e)$. Then by (7)

$$
B_{L} x^{-1} B y B_{L}=x^{-1} \cdot x B_{L} x^{-1} \cdot B \cdot y B_{L} y^{-1} \cdot y \subseteq x^{-1} B y .
$$

Let $x, y \in D(e), z, w \in W(e), a \in x^{-1} B y \cap B^{-} w B$ be such that $\xi(a) \in B_{L} z B_{L}$. Then $B_{L} z B_{L}=B_{L} \xi(a) B_{L}$. Let $b_{1}, b_{2} \in B_{L}$ be such that $b_{1} \xi(a) b_{2} \in B_{L}^{-} w B_{L}$. Now by (15), $b_{1} a b_{2} \in x^{-1} B y$. Also $a=v \xi(a) u$ for some $v \in U_{I}^{-}, u \in U_{I}$. So

$$
\begin{aligned}
b_{1} a b_{2} & =b_{1} v \xi(a) u b_{2} \\
& =b_{1} v b_{1}^{-1} \cdot b_{1} \xi(a) b_{2} \cdot b_{2}^{-1} u b_{2} \\
& \in U_{I}^{-} B_{L}^{-} w B_{L} U_{I} \\
& =B^{-} w B
\end{aligned}
$$

and $\xi\left(b_{1} a b_{2}\right)=b_{1} \xi(a) b_{2}$. Thus, for $x, y \in D(e)$ and $z, w \in W(e)$,

$$
B_{L} z B_{L} \cap \xi\left(x^{-1} B y \cap B^{-} w B\right) \neq \varnothing
$$

implies

$$
B_{L} z B_{L} \cap B_{L}^{-} w B_{L} \subseteq \xi\left(x^{-1} B y \cap B^{-} w B\right) .
$$

For $z, w \in W(e)$, let

$$
X_{z, w}=x^{-1} B y \cap \xi^{-1}\left(B_{L} z B_{L} \cap B_{L}^{-} w B_{L}\right) .
$$

Then

$$
X_{z, w} \neq \varnothing \quad \text { implies } \quad \xi\left(X_{z, w}\right)=B_{L} z B_{L} \cap B_{L}^{-} w B_{L} .
$$

Suppose $X_{z, w} \neq \varnothing$, and let $a \in X_{z, w}$. Then $\xi(a)=b_{1} \dot{z} b_{2}$ for some $b_{1}, b_{2} \in B_{L}$. Let $c \in X_{z, w}$ be such that $\xi(a)=\xi(c)$. Then for some $v \in U_{I}^{-}, u \in U_{I}$,

$$
\begin{aligned}
c & =v b_{1} \dot{z} b_{2} u \\
& =b_{1} \cdot b_{1}^{-1} v b_{1} \cdot \dot{z} \cdot b_{2} u b_{2}^{-1} \cdot b_{2} \\
& =b_{1} v_{1} \dot{z} u_{1} b_{2} \quad \text { with } v_{1}=b_{1}^{-1} v b_{1} \in U_{I}^{-}, u_{1}=b_{2} u b_{2}^{-1} \in U_{I} .
\end{aligned}
$$

Also by (15)

$$
v_{1} \dot{z} u_{1}=b_{1}^{-1} c b_{2}^{-1} \in b_{1}^{-1} x^{-1} B y b_{2}^{-1} \subseteq x^{-1} B y .
$$

Thus

$$
v_{1} \dot{z} u_{1} \in x^{-1} B y \cap U_{I}^{-} \dot{z} U_{I} .
$$

Conversely, if $v_{1} \in U_{I}^{-}, u_{1} \in U_{I}$ with $v_{1} \dot{z} u_{1} \in x^{-1} B y$, then by (15),

$$
c=b_{1} v_{1} \dot{z} u_{1} b_{2}=b_{1} v_{1} b_{1}^{-1} \cdot b_{1} \dot{z} b_{2} \cdot b_{2}^{-1} u_{1} b_{2} \in x^{-1} B y \cap U_{I}^{-} \xi(a) U_{I} .
$$


Hence, $c \in X_{z, w}$ and $\xi(a)=\xi(c)$. Thus each fibre of $\xi$ restricted to $X_{z, w}$ has cardinality $\left|x^{-1} B y \cap U_{I}^{-} \dot{z} U_{I}\right|$. Hence by (16)

$$
X_{z, w} \neq \varnothing \quad \text { implies } \quad\left|X_{z, w}\right|=\left|B_{L} z B_{L} \cap B_{L}^{-} w B_{L}\right| \cdot\left|U_{I}^{-} \dot{z} U_{I} \cap x^{-1} B y\right| .
$$

Now suppose $B_{L} z B_{L} \cap B_{L}^{-} w B_{L} \neq \varnothing$ and $U_{I}^{-} \dot{z} U_{I} \cap x^{-1} B y \neq \varnothing$. Then for some $v \in U_{I}^{-}$and $u \in U_{I}$ we have $v \dot{z} u \in x^{-1} B y$. Also for some $b \in B_{L}, b \dot{z} \in B_{L}^{-} w B_{L}$. Then by (15)

$$
b v \dot{z} u=b v b^{-1} \cdot b \dot{z} \cdot u \in X_{z, w} .
$$

So $X_{z, w} \neq \varnothing$. Hence in all cases

$$
\left|X_{z, w}\right|=\left|B_{L} z B_{L} \cap B_{L}^{-} w B_{L}\right| \cdot\left|U_{I}^{-} \dot{z} U_{I} \cap x^{-1} B y\right| .
$$

Thus

$$
\begin{aligned}
\left|x^{-1} B y \cap B^{-} w B\right| & =\sum_{z \in W(e)}\left|X_{z, w}\right| \\
& =\sum_{z \in W(e)}\left|B_{L} z B_{L} \cap B_{L}^{-} w B_{L}\right| \cdot\left|U_{I}^{-} \dot{z} U_{I} \cap x^{-1} B y\right| .
\end{aligned}
$$

Now by $[12],[5]$

$$
\left|B_{L} z B_{L} \cap B_{L}^{-} w B_{L}\right|=R_{w, z}\left|B_{L}\right|
$$

and by $(15)$

$$
\begin{aligned}
& \left|B_{L}\right| \cdot\left|U_{I}^{-} \dot{z} U_{I} \cap x^{-1} B y\right|=\left|U_{I}^{-} B_{L} z U_{I} \cap x^{-1} B y\right| \\
& \quad=q^{-\ell(z)}\left|U_{I}^{-} B_{L} z B \cap x^{-1} B y\right| .
\end{aligned}
$$

Hence

$$
\begin{array}{r}
\left|B_{L} z B_{L} \cap B_{L}^{-} w B_{L}\right| \cdot\left|U_{I}^{-} \dot{z} U_{I} \cap x^{-1} B y\right| \\
=q^{-\ell(z)} R_{w, z}\left|U_{I}^{-} B_{L} z B \cap x^{-1} B y\right| .
\end{array}
$$

By [12], the inverse of the matrix $\left(q^{-\ell(z)} R_{w, z}\right)$ is $\left((-1)^{\ell(z)+\ell(w)} q^{\ell(w)} R_{w, z}\right)$. Thus by (17), (18)

$$
\left|U_{I}^{-} B_{L} w B \cap x^{-1} B y\right|=q^{\ell(w)} \cdot \sum_{z \in W(e)}(-1)^{\ell(z)+\ell(w)} R_{w, z}\left|B^{-} z B \cap x^{-1} B y\right| .
$$

Let $z \in W(e), U_{L}^{-}=U^{-} \cap B_{L}^{-}, U_{L}=U \cap B_{L}$, and

$$
\begin{aligned}
& X=\left\{v \in U_{L}^{-} \mid z v z^{-1} \in U_{L}^{-}\right\}, \\
& Y=\left\{v \in U_{L}^{-} \mid z v z^{-1} \in U_{L}\right\} .
\end{aligned}
$$

Then

$$
\begin{aligned}
x^{-1} B y \cap B^{-} z B & =x^{-1} B y \cap U_{I}^{-} U_{L}^{-} z B \\
& =x^{-1} B y \cap U_{I}^{-} z X B \\
& =x^{-1} B y \cap z U_{I}^{-} X B
\end{aligned}
$$


and

$$
\begin{aligned}
\left|x^{-1} B y \cap z B^{-} B\right| & =\left|x^{-1} B y \cap z U_{I}^{-} U_{L}^{-} B\right| \\
& =\left|x^{-1} B y \cap U_{I}^{-} z U_{L}^{-} B\right| \\
& =\left|x^{-1} B y \cap U_{I}^{-} z Y X B\right| \\
& =\left|x^{-1} B y \cap U_{I}^{-} z Y z^{-1} \cdot z X B\right| \\
& =\left|x^{-1} B y \cap z Y z^{-1} \cdot U_{I}^{-} z X B\right| \\
& =\left|z Y z^{-1}\right| \cdot\left|x^{-1} B y \cap U_{I}^{-} z X B\right|, \quad \text { by (15) } \\
& =q^{\ell(z)}\left|x^{-1} B y \cap z U_{I}^{-} X B\right| \\
& =q^{\ell(z)}\left|x^{-1} B y \cap B^{-} z B\right| .
\end{aligned}
$$

Hence

$$
\left|x^{-1} B y \cap z B^{-} B\right|=q^{\ell(z)}\left|x^{-1} B y \cap B^{-} z B\right| .
$$

By [19, Theorem 2.4]

$$
\begin{aligned}
\left|x^{-1} B y \cap z B^{-} B\right| & =\left|z^{-1} x^{-1} B y \cap B^{-} B\right| \\
& =|B| \cdot q^{\ell(x z)-\ell(y)} R_{x z, y} .
\end{aligned}
$$

Hence, by (20)

$$
\left|x^{-1} B y \cap B^{-} z B\right|=|B| \cdot q^{\ell(x)-\ell(y)} R_{x z, y} .
$$

So by (14), (19), (21),

$$
Q_{x, y}=\sum_{w \in W(e)} q^{\ell(w)} \sum_{z \in W(e)}(-1)^{\ell(z)+\ell(w)} q^{\ell(x)-\ell(y)} R_{w, z} R_{x z, y} \cdot A_{w} .
$$

Now consider the generic monoid Hecke algebra $\mathcal{H}(R)$. Fix $e \in \Lambda$ and let

$$
\mathcal{I}=\sum_{\substack{\sigma \in W f W \\ f<e}} \mathbb{Z}\left[q^{1 / 2}, q^{1 / 2}\right] A_{\sigma} .
$$

Then the orbit Hecke algebra

$$
\mathcal{H}(e)=\sum_{\sigma \in W e W} \mathbb{Z}\left[q^{1 / 2}, q^{-1 / 2}\right] A_{\sigma}
$$

is an ideal of $\mathcal{H}(R) / \mathcal{I}$. Again we call

$$
\widehat{\mathcal{H}}(e)=\mathcal{H}(e)+\mathcal{H}(W)
$$

the augmented orbit Hecke algebra.

Since (13), (22) are valid for all $q$, they are valid in $\widehat{\mathcal{H}}(e)$. Now in $\widehat{\mathcal{H}}(e)$,

$$
\begin{aligned}
Q_{x, y} & =q^{\ell(x)-\ell(y)} \sum_{z \in W(e)} R_{x z, y} \sum_{w \in W(e)}(-1)^{\ell(z)+\ell(w)} q^{\ell(w)} R_{w, z} A_{w} \\
& =q^{\ell(x)-\ell(y)} \sum_{z \in W(e)} R_{x z, y} \sum_{w \in W(e)} q^{\ell(z)} \bar{R}_{w, z} A_{w} \\
& =q^{\ell(x)-\ell(y)} \sum_{z \in W(e)} R_{x z, y} \bar{A}_{z}, \quad \text { by }(5) .
\end{aligned}
$$


By [19, Theorem 3.1], the unity of $\mathcal{H}(e)$ is obtained by inverting the matrix $\left(Q_{x, y}\right)$. Now for $y \in D(e)$ we have

$$
\begin{aligned}
A_{y} & =q^{-\ell(y)} \sum_{w \in W} R_{w, y} \bar{A}_{w}, \quad \text { by }(5) \\
& =q^{-\ell(y)} \sum_{x \in D(e)} \sum_{z \in W(e)} R_{x z, y} \bar{A}_{x z} \\
& =q^{-\ell(y)} \sum_{x \in D(e)} \bar{A}_{x} \sum_{z \in W(e)} R_{x z, y} \bar{A}_{z}, \quad \text { by }(3) \\
& =\sum_{x \in D(e)} q^{-\ell(x)} \bar{A}_{x} Q_{x, y} .
\end{aligned}
$$

So, for $x \in D(e)$,

$$
\bar{A}_{x}=\sum_{w \in D(e)} q^{\ell(w)} A_{w} \bar{Q}_{w, x}
$$

Hence

$$
\begin{aligned}
A_{y} & =\sum_{x \in D(e)} \sum_{w \in D(e)} q^{\ell(w)-\ell(x)} A_{w} \bar{Q}_{w, x} Q_{x, y} \\
& =\sum_{w \in D(e)} A_{w}\left(\sum_{x \in D(e)} q^{\ell(w)-\ell(x)} \bar{Q}_{w, x} Q_{x, y}\right) .
\end{aligned}
$$

Thus, for $y, w \in D(e)$,

$$
\sum_{x \in D(e)} q^{\ell(w)-\ell(x)} \bar{Q}_{w, x} Q_{x, y}=\delta_{w, y} A_{e}
$$

Hence $\left(q^{\ell(x)-\ell(y)} \bar{Q}_{x, y}\right)$ is the inverse of $\left(Q_{x, y}\right)$. Now

$$
q^{\ell(x)-\ell(y)} \bar{Q}_{x, y}=\sum_{z \in W(e)} \bar{R}_{x z, y} A_{z} .
$$

Hence by [19, Theorem 3.1] the unity $\hat{e}$ of the orbit algebra $\mathcal{H}(e)$ is given by

$$
\hat{e}=\sum_{\substack{x, y \in D(e) \\ z \in W(e)}} \bar{R}_{x z, y} A_{x z e y^{-1}} .
$$

For $\sigma=x e y^{-1} \in W e W$ in standard form, let

$$
R_{\sigma}=\sum_{z \in W_{e}} R_{x z, y} .
$$

Remark 3.1. (i) If $W_{e}=W_{K}, K \subseteq S$, then by Deodhar [6], $R_{\sigma}=R_{x, y}^{K}$. We also note that the condition $x z \leq y$ has been studied by Deodhar [7].

(ii) By (2), $R_{\sigma} \neq 0$ if and only if $\sigma$ is triangular.

By (24), we now have

Theorem 3.2. The unity $\hat{e}$ of the generic orbit Hecke algebra $\mathcal{H}(e)$ is given by

$$
\hat{e}=\sum_{\sigma \in W e W} \bar{R}_{\sigma} A_{\sigma}=\sum_{\sigma \in W e W} q^{-\ell(\sigma)} \bar{R}_{\sigma} T_{\sigma} .
$$


Now consider $\hat{e}$ as an element of $\mathcal{H}(R)$ and let

$$
\tilde{e}=\hat{e} \cdot \prod_{\substack{f \in \Lambda \\ f<e}}(1-\hat{f}) .
$$

Using the order on $\Lambda$ and proceeding by induction, beginning with the least element, we have

Theorem 3.3. (i) The elements $\tilde{e}, e \in \Lambda$ are independent of the order of the product in (26).

(ii) $\tilde{e}(e \in \Lambda)$ form an orthogonal set of central idempotents of $\mathcal{H}(R)$, and hence $\mathcal{H}(R)=\bigoplus_{e \in \Lambda} \tilde{e} \mathcal{H}(R)$.

(iii) The map $A_{\sigma} \rightarrow \tilde{e} A_{\sigma}, \sigma \in W e W$, extends to an isomorphism between $\mathcal{H}(e)$ and $\tilde{e} \mathcal{H}(R)$.

(iv) If $\mathcal{I}$ is as in (23), then the natural homomorphism from $\mathcal{H}(R)$ to $\mathcal{H}(R) / \mathcal{I}$ restricts to an isomorphism between $\tilde{e} \mathcal{H}(R)$ and $\mathcal{H}(e)$. This isomorphism is the inverse of the isomorphism in (iii).

Remark 3.4. (i) By Theorem 3.2 and [19, Theorem 3.1] we have an explicit isomorphism betwen $\mathcal{H}(e)$ and $M_{d}\left(\mathcal{H}\left(W_{K}\right)\right)$, where $d=|D(e)|$ and $W(e)=W_{e} \times W_{K}$, $K \subseteq S$. We therefore have a sequence of homomorphisms

$$
\mathcal{H}(R) \rightarrow \mathcal{H}(R) / I \rightarrow \mathcal{H}(e) \rightarrow M_{d}\left(\mathcal{H}\left(W_{K}\right)\right)
$$

Upon specializing $q$, we see that an irreducible representation of degree $n$ of $\mathcal{H}\left(W_{K}\right)$ yields explicitly an irreducible representation of degree $n d$ of $H_{\mathbb{C}}(M, B)$. Moreover every irreducible representation of $H_{\mathbb{C}}(M, B)$ is obtained in this manner for some $e \in \Lambda$.

(ii) Let $\Lambda=\mathbb{Z}\left[q^{1 / 2}, q^{-1 / 2}\right]$. Then as in Theorem 3.3 (but much more easily) $\Gamma[R]$ is explicitly isomorphic to a direct sum of orbit algebras $\Gamma_{0}\left[J^{0}\right]$. For $J=W e W$, the unity $\underline{e}$ of $\Gamma_{0}\left[J^{0}\right]$ is given by

$$
\underline{e}=\sum_{x \in D(e)} x e x^{-1} .
$$

If $d=|D(e)|$, then $\Gamma_{0}\left[J^{0}\right]$ is isomorphic to $M_{d}\left(\Gamma\left[W_{K}\right]\right)$, where $W(e)=W_{e} \times W_{K}$, $K \subseteq S$. Now by Lusztig [13] (or see [3]) we have an explicit isomorphism between $Q\left(q^{1 / 2}\right)\left[W_{K}\right]$ and $\mathcal{H}\left(W_{K}\right) \otimes_{\Gamma} Q\left(q^{1 / 2}\right)$. Combining with (i), we have an explicit but very complicated isomorphism between $\mathcal{H}(R) \otimes_{\Gamma} Q\left(q^{1 / 2}\right)$ and $Q\left(q^{1 / 2}\right)[R]$, solving a problem posed by Solomon [28].

\section{Involution}

We wish to extend the Kazhdan-Lusztig involution (4) on $\mathcal{H}(W)$ to $\mathcal{H}(R)$. By Theorem 3.3 it suffices to do this for the augmented orbit Hecke algebras $\widehat{\mathcal{H}}(e)$. We accomplish this by considering the order (1) on $W e W$ and remembering that, by $(5), \bar{A}_{x},(x \in W)$ is a linear combination of $A_{y}, y \leq x$. If $\sigma=x e y^{-1}$ in standard form, then, by (1),

$$
\sigma \leq e \text { if and only if } \quad x \in W(e) \text { and } x \leq y .
$$


Theorem 4.1. There is a unique extension of the involution on $\mathcal{H}(W)$ to $\widehat{\mathcal{H}}(e)$ such that

$$
\bar{A}_{e}=\sum_{\substack{z \in W(e) \\ y \in D(e)}} \bar{R}_{z, y} A_{z e y^{-1}}
$$

If $\sigma=$ set $^{-1} \in W e W$ in standard form, then

$$
\bar{A}_{\sigma}=q^{-\ell(t)} \bar{A}_{s} \cdot \sum_{\substack{z \in W(e) \\ y \in D(e)}} \bar{R}_{t z, y} A_{z e y^{-1}} .
$$

Proof. Let $w_{0}$ and $v_{0}$ denote the longest element in $W$ and $W(e)$, respectively. Then $u_{0}=w_{0} v_{0}$ is the longest element in $D(e)$. Also

$$
\ell\left(x w_{0}\right)=\ell\left(w_{0} x\right)=\ell\left(w_{0}\right)-\ell(x) \quad \text { for all } x \in W .
$$

Hence by (3)

$$
A_{x w_{0}}=\bar{A}_{x} A_{w_{0}} \quad \text { and } \quad A_{w_{0} x}=A_{w_{0}} \bar{A}_{x} \quad \text { for all } x \in W .
$$

If $\sigma=$ set $^{-1}$ in standard form, then

$$
\begin{aligned}
& \bar{A}_{\sigma}=\bar{A}_{s} \cdot q^{-\ell(t)} \cdot \sum_{\substack{z \in W(e) \\
y \in D(e)}} \bar{R}_{t z, y} A_{z e y^{-1}} \\
& =\bar{A}_{s} \cdot q^{-\ell(t)} \cdot \sum_{\substack{z \in W(e) \\
y \in D(e)}} \bar{R}_{z^{-1} t^{-1}, y^{-1}} A_{z e y^{-1}} \\
& =\bar{A}_{s} \cdot q^{-\ell(t)} \cdot \sum_{\substack{z \in W(e) \\
y \in D(e)}} \bar{R}_{v_{0} z \cdot z^{-1} t^{-1}, v_{0} z \cdot y^{-1}} A_{z e y^{-1}}, \quad \text { by }(28) \\
& =\bar{A}_{s} \cdot q^{-\ell(t)} \sum_{\substack{z \in W(e) \\
y \in D(e)}} \bar{R}_{v_{0} t^{-1}, v_{0} z y^{-1}} A_{z e y^{-1}} \\
& =\bar{A}_{s} \cdot q^{-\ell(t)} A_{e} \cdot \sum_{\substack{z \in W(e) \\
y \in D(e)}} \bar{R}_{v_{0} t^{-1}, v_{0} z y^{-1}} A_{z} \bar{A}_{y^{-1}}, \quad \text { by }(9),(10) \\
& =\bar{A}_{s} \cdot q^{-\ell(t)} A_{e} \cdot \sum_{\substack{z \in W(e) \\
y \in D(e)}} \bar{R}_{v_{0} t^{-1}, z y^{-1}} A_{v_{0} z} \bar{A}_{y^{-1}} \\
& =\bar{A}_{s} \cdot q^{-\ell(t)} A_{e} A_{v_{0}} \cdot \sum_{\substack{z \in W(e) \\
y \in D(e)}} \bar{R}_{v_{0} t^{-1}, z y^{-1}} \bar{A}_{z} \bar{A}_{y^{-1}}, \quad \text { by }(29) \\
& =\bar{A}_{s} \cdot q^{-\ell(t)} A_{e} A_{v_{0}} \cdot \sum_{x \in W} \bar{R}_{v_{0} t^{-1}, x} \bar{A}_{x}, \quad \text { by }(3) \\
& =\bar{A}_{s} \cdot q^{-\ell(t)} A_{e} A_{v_{0}} \cdot \sum_{x \in W} \bar{R}_{v_{0} t^{-1}, w_{0} x} \bar{A}_{w_{0} x} \\
& =\bar{A}_{s} \cdot q^{-\ell(t)} A_{e} A_{v_{0}} \cdot \sum_{x \in W} \bar{R}_{x, u_{0} t^{-1}} \bar{A}_{w_{0} x} \\
& =\bar{A}_{s} \cdot q^{-\ell(t)} A_{e} A_{v_{0}} \bar{A}_{w_{0}} \cdot \sum_{x \in W} \bar{R}_{x, u_{0} t^{-1}} \bar{A}_{x}, \quad \text { by }(29)
\end{aligned}
$$




$$
\begin{aligned}
& =\bar{A}_{s} \cdot q^{-\ell(t)} A_{e} A_{v_{0}} \bar{A}_{w_{0}} \cdot q^{-\ell(e)+\ell(t)} \bar{A}_{u_{0} t^{-1}}, \quad \text { by }(5),(28) \\
& =q^{-\ell(e)} \bar{A}_{s} A_{e} A_{v_{0}} \bar{A}_{w_{0}} \bar{A}_{u_{0} t^{-1}} \\
& =q^{-\ell(e)} \bar{A}_{s} A_{e} \bar{A}_{u_{0}^{-1}} \bar{A}_{u_{0} t^{-1}}, \quad \text { by }(29) \\
& =q^{-\ell(e)} \bar{A}_{s} A_{e} \bar{A}_{u_{0}^{-1}} \bar{A}_{w_{0}} A_{v_{0} t^{-1}}, \quad \text { by }(29) \\
& =q^{-\ell(e)} \bar{A}_{s} A_{e} \bar{A}_{u_{0}^{-1}} \bar{A}_{w_{0}} A_{v_{0}} A_{t^{-1}}, \quad \text { by }(3) \\
& =q^{-\ell(e)} \bar{A}_{s} A_{e} \bar{A}_{u_{0}^{-1}} \bar{A}_{u_{0}} A_{t^{-1}}, \quad \text { by }(29) .
\end{aligned}
$$

Hence,

$$
\bar{A}_{\sigma}=q^{-\ell(e)} \bar{A}_{s} A_{e} \bar{A}_{u_{0}^{-1}} \bar{A}_{u_{0}} A_{t^{-1}} .
$$

In particular,

$$
\bar{A}_{e}=q^{-\ell(e)} A_{e} \bar{A}_{u_{0}^{-1}} \bar{A}_{u_{0}} .
$$

By (13), (14), (22), we see that

$$
A_{e x^{-1}} A_{y e}=0 \quad \text { if } x, y \in D(e), y<x .
$$

Hence, by (3), (5), (9), (10)

$$
A_{e} \bar{A}_{x^{-1}} \bar{A}_{x} A_{e}=q^{\ell(x)} A_{e} \quad \text { for } x \in D(e)
$$

and

$$
A_{e} \bar{A}_{x^{-1}} \bar{A}_{y} A_{e}=0 \quad \text { if } x \in D(e), y \in W, y<x .
$$

By (31), (32)

$$
\bar{A}_{e} \cdot \bar{A}_{e}=\bar{A}_{e}
$$

By (29) we see that for all $x \in W$,

$$
A_{w_{0}} A_{x} \bar{A}_{w_{0}}=A_{w_{0}} \bar{A}_{x w_{0}}=A_{w_{0} x w_{0}}=\bar{A}_{w_{0} x} A_{w_{0}}=\bar{A}_{w_{0}} A_{x} A_{w_{0}} .
$$

Hence for all $x \in W$,

$$
A_{w_{0} x w_{0}}=A_{w_{0}} A_{x} \bar{A}_{w_{0}}=\bar{A}_{w_{0}} A_{x} A_{w_{0}} \quad \text { and } \quad A_{w_{0}}^{2} A_{x}=A_{x} A_{w_{0}}^{2} .
$$

By (29), (35) we see that for all $z \in W(e)$,

$$
\begin{aligned}
\bar{A}_{u_{0}^{-1}} \bar{A}_{u_{0}} A_{z} & =A_{v_{0}} \bar{A}_{w_{0}} \bar{A}_{w_{0}} A_{v_{0}} A_{z}=\bar{A}_{w_{0}}^{2} A_{v_{0}}^{2} A_{z} \\
& =A_{z} \bar{A}_{w_{0}}^{2} A_{v_{0}}^{2}=A_{z} A_{v_{0}} \bar{A}_{w_{0}} \bar{A}_{w_{0}} A_{v_{0}}=A_{z} \bar{A}_{u_{0}^{-1}} \bar{A}_{u_{0}} .
\end{aligned}
$$

Hence by (5), (10), (31)

$$
\bar{A}_{e} A_{z}=A_{z} \bar{A}_{e}, \bar{A}_{e} \bar{A}_{z}=\bar{A}_{z} \bar{A}_{e} \quad \text { for all } z \in W(e) .
$$

If $x \in D(e)$, then by (28),

$$
\begin{aligned}
\ell\left(u_{0} x^{-1}\right) & =\ell\left(w_{0} v_{0} x^{-1}\right)=\ell\left(w_{0}\right)-\ell\left(v_{0} x^{-1}\right) \\
& =\ell\left(w_{0}\right)-\ell\left(v_{0}\right)-\ell(x)=\ell\left(u_{0}\right)-\ell(x) .
\end{aligned}
$$

So, by (3), $A_{u_{0}}=A_{u_{0} x^{-1}} A_{x}$. Hence

$$
\bar{A}_{e} A_{x^{-1}}=A_{e} \bar{A}_{u_{0}^{-1}} \bar{A}_{u_{0} x^{-1}} \quad \text { for all } x \in D(e) \text {. }
$$

So, by (33),

$$
\bar{A}_{e} A_{x^{-1}} \bar{A}_{e}=0 \quad \text { for } x \in D(e), x \neq 1 .
$$


So, by (3), (36),

$$
\bar{A}_{e} A_{x} \bar{A}_{e}=0 \quad \text { for all } x \in W \backslash W(e) .
$$

By (3), (5), (10), (33), $A_{e} \bar{A}_{x} A_{e}=0$ for $x \in W \backslash W(e)$. Hence, by (5), (34), (36),

$$
\overline{A_{e} A_{x} A_{e}}=\bar{A}_{e} \bar{A}_{x} \bar{A}_{e} \quad \text { for all } x \in W .
$$

By (3), (5), (30), (31), (36),

$$
\overline{A_{x} A_{\sigma}}=\bar{A}_{x} \bar{A}_{\sigma}, \overline{A_{\sigma} A_{x}}=\bar{A}_{\sigma} \bar{A}_{x} \quad \text { for all } x \in W, \sigma \in W e W .
$$

It follows from (5), (9), (37) that ${ }^{-}: \widehat{\mathcal{H}}(e) \rightarrow \widehat{\mathcal{H}}(e)$ is a homomorphism. To complete the proof we need to show that $\overline{\bar{A}}_{e}=A_{e}$. Now, by (5), (31),

$$
\begin{aligned}
\overline{\bar{A}}_{e} & =q^{\ell(e)} \bar{A}_{e} A_{u_{0}^{-1}} A_{u_{0}} \\
& =q^{\ell(e)} q^{-\ell(e)} A_{e} \bar{A}_{u_{0}^{-1}} \bar{A}_{u_{0}} A_{u_{0}^{-1}} A_{u_{0}} \\
& =A_{e} .
\end{aligned}
$$

This completes the proof.

If $x, z \in W$, then we see by induction on $\ell(z)$ that

$$
A_{x} A_{z} \in \sum_{\substack{y \leq x \\ w \leq z \\ \ell(y w)=\ell(y)+\ell(w)}} \mathbb{Z}\left[q^{-1}\right] A_{y w} .
$$

By (3), (5), (38) and Theorem 4.1, we have

Corollary 4.2. Let $\sigma \in W e W$. Then there exist $R_{\theta, \sigma} \in \mathbb{Z}[q], \theta \in W e W$, such that, in $\widehat{\mathcal{H}}(e)$,

(i) $\bar{A}_{\sigma}=q^{\ell(\sigma)-\ell(e)} \sum_{\theta \in W e W} \bar{R}_{\theta, \sigma} A_{\theta}$,

(ii) $R_{\theta, \sigma} \neq 0$ only if $\theta \leq \sigma$,

(iii) $R_{\sigma, \sigma}=1$.

Problem 4.3. Determine the polynomials $R_{\theta, \sigma}$ explicitly for $\sigma, \theta \in W e W$. Does $\theta \leq \sigma$ imply $R_{\theta, \sigma} \neq 0$ ?

When $W(e)=W_{e}$, Theorem 4.1 can be simplified by the use of the relative $R$-polynomials of Deodhar [6, Proposition 2.12].

Corollary 4.4. Suppose $W(e)=W_{e}=W_{I}, I \subseteq S$. Let $\sigma=$ set $^{-1} \in W e W$ in standard form. Then $s, t \in D(e)$ and

$$
\bar{A}_{\sigma}=q^{\ell(s)-\ell(t)} \sum_{x, y \in D(e)} \bar{R}_{x, s}^{I} \bar{R}_{t, y}^{I} A_{x e y^{-1}} .
$$

In [12], Kazhdan and Lusztig introduced the now-famous polynomials $P_{x, y} \in \mathbb{Z}[q]$ to define a basis

$$
C_{y}=(-1)^{\ell(y)} q^{\ell(y) / 2} \sum_{x \in W}(-1)^{\ell(x)} \bar{P}_{x, y} A_{x}, \quad y \in W,
$$

of the $\mathbb{Z}\left[q^{1 / 2}, q^{-1 / 2}\right]$-algebra $\mathcal{H}(W)$ such that $\bar{C}_{y}=C_{y}$ for all $y \in W$. We refer to $[10$, Chapter 7$]$ for details. 
Let $e \in \Lambda$, and let $w_{0}$ and $v_{0}$ denote the longest element of $W$ and $W(e)$, respectively. For $\sigma=$ set $^{-1}$ in standard form, let

$$
C_{\sigma}=(-1)^{\ell(t)} q^{-\ell(t) / 2} C_{s} \cdot \sum_{\substack{y \in D(e) \\ z \in W(e)}}(-1)^{\ell(y z)} \bar{P}_{w_{0} y z v_{0}, w_{0} t v_{0}} A_{z^{-1} e y^{-1}} .
$$

Theorem 4.5. Let $e \in \Lambda$. Then $\bar{C}_{\sigma}=C_{\sigma}$ for all $\sigma \in W e W$, and they form a $\mathbb{Z}\left[q^{1 / 2}, q^{-1 / 2}\right]$-basis of $\mathcal{H}(e)$.

Proof. Let $\sigma=$ set $^{-1}$ in standard form. Then it is easy to see that

$$
q^{(\ell(t)-\ell(s)) / 2} C_{\sigma}-A_{\sigma}
$$

is a $\mathbb{Z}\left[q^{1 / 2}, q^{-1 / 2}\right]$-linear combination of $A_{\theta}$ such that if $\theta=x e y^{-1}$ in standard form, then either $y<t$ or else $y=t$ and $s<x$. It follows that $C_{\sigma}(\sigma \in W e W)$ form a $\mathbb{Z}\left[q^{1 / 2}, q^{-1 / 2}\right]$-basis of $\mathcal{H}(e)$. Thus by (39) it suffices to show that, for all $t \in D(e)$, $\bar{C}_{e t^{-1}}=C_{e t^{-1}}$. So fix $t \in D(e)$. Let $u_{0}=w_{0} v_{0} \in D(e)$. Then, by (28),

$$
w_{0} y v_{0} \in D(e), \quad \ell\left(w_{0} y v_{0}\right)=\ell\left(u_{0}\right)-\ell(y) \quad \text { for all } y \in D(e) .
$$

For $y \in D(e)$ let

$$
\mathcal{P}_{t, y}=q^{-\ell(t) / 2} \sum_{z \in W(e)}(-1)^{\ell(y z)} \bar{P}_{w_{0} y z v_{0}, w_{0} t v_{0}} A_{z^{-1}} .
$$

So, by (40),

$$
C_{e t^{-1}}=(-1)^{\ell(t)} \sum_{y \in D(e)} \mathcal{P}_{t, y} A_{e y^{-1}} .
$$

Hence by (27)

$$
\bar{C}_{e t^{-1}}=(-1)^{\ell(t)} \sum_{y \in D(e)} \overline{\mathcal{P}}_{t, y} \cdot \sum_{\substack{x \in D(e) \\ z \in W(e)}} q^{-\ell(y)} \bar{R}_{y z, x} A_{z e x^{-1}} .
$$

Let

$$
\mathcal{R}_{y, x}=q^{-\ell(y)} \sum_{z \in W(e)} \bar{R}_{y z, x} A_{x} .
$$

So

$$
\bar{C}_{e t^{-1}}=(-1)^{\ell(t)} \sum_{x, y \in D(e)} \overline{\mathcal{P}}_{t, y} \mathcal{R}_{y, x} A_{e x^{-1}} .
$$

Thus we need to show that, for all $x \in D(e)$,

$$
\mathcal{P}_{t, x}=\sum_{y \in D(e)} \overline{\mathcal{P}}_{t, y} \mathcal{R}_{y, x} .
$$


Now by (3), (39), (41),

$$
\begin{aligned}
& C_{v_{0} t^{-1} w_{0}}=(-1)^{\ell\left(t u_{0}\right)} q^{\left(\ell\left(u_{0}\right)-\ell(t)\right) / 2} \sum_{x \in W}(-1)^{\ell(x)} \bar{P}_{x, w_{0} t v_{0}} A_{x^{-1}} \\
& =(-1)^{\ell\left(t u_{0}\right)} q^{\left(\ell\left(u_{0}\right)-\ell(t)\right) / 2} \sum_{y \in D(e)}\left[\sum_{z \in W(e)}(-1)^{\ell\left(y u_{0} z\right)} \bar{P}_{w_{0} y v_{0} z, w_{0} t v_{0}} A_{z^{-1}}\right] \cdot A_{v_{0} y^{-1} w_{0}} \\
& =(-1)^{\ell(t)} q^{\ell\left(u_{0}\right) / 2} \sum_{y \in D(e)}\left[q^{-\ell(t) / 2} \sum_{z \in W(e)}(-1)^{\ell(y z)} \bar{P}_{w_{0} y v_{0} z, w_{0} t v_{0}} A_{z^{-1}}\right] \cdot A_{v_{0} y^{-1} w_{0}} .
\end{aligned}
$$

Substituting $v_{0} z v_{0}$ for $z$ and using (35), (42), we see that

$$
C_{v_{0} t^{-1} w_{0}}=(-1)^{\ell(t)} q^{\ell\left(u_{0}\right) / 2} \sum_{y \in D(e)} \bar{A}_{v_{0}} \mathcal{P}_{t, y} A_{v_{0}} \cdot A_{v_{0} y^{-1} w_{0}}
$$

So

$$
\bar{C}_{v_{0} t^{-1} w_{0}}=(-1)^{\ell(t)} q^{-\ell\left(u_{0}\right) / 2} \sum_{y \in D(e)} A_{v_{0}} \overline{\mathcal{P}}_{t, y} \bar{A}_{v_{0}} \cdot \bar{A}_{v_{0} y^{-1} w_{0}} .
$$

By (3), (5), (41),

$$
\begin{aligned}
& \bar{A}_{v_{0} y^{-1} w_{0}}=q^{\ell\left(u_{0}\right)-\ell(y)} \sum_{\substack{x \in D(e) \\
z \in W(e)}} \bar{R}_{w_{0} x v_{0} z, w_{0} y v_{0}} A_{z^{-1}} A_{v_{0} x^{-1} w_{0}} \\
& =q^{\ell\left(u_{0}\right)-\ell(y)} \sum_{\substack{x \in D(e) \\
z \in W(e)}} \bar{R}_{y v_{0}, x v_{0} z} A_{z}^{-1} A_{v_{0} x^{-1} w_{0}} \\
& =q^{\ell\left(u_{0}\right)-\ell(y)} \sum_{\substack{x \in D(e) \\
z \in W(e)}} \bar{R}_{y v_{0} z^{-1} v_{0}, x} A_{z^{-1}} A_{v_{0} x^{-1} w_{0}}, \\
& \text { since } \ell\left(y v_{0}\right)=\ell\left(y v_{0} z^{-1} v_{0}\right)+\ell\left(v_{0} z\right) \\
& \text { and } \ell\left(x v_{0} z\right)=\ell(x)+\ell\left(v_{0} z\right) \\
& =q^{\ell\left(u_{0}\right)-\ell(y)} \sum_{\substack{x \in D(e) \\
z \in W(e)}} \bar{R}_{y z, x} A_{v_{0} z v_{0}} A_{v_{0} x^{-1} w_{0}} \\
& =q^{\ell\left(u_{0}\right)-\ell(y)} \sum_{x \in D(e)} A_{v_{0}}\left[\sum_{z \in W(e)} \bar{R}_{y z, x} A_{z}\right] \bar{A}_{v_{0}} A_{v_{0} x^{-1} w_{0}}, \quad \text { by (35) } \\
& =q^{\ell\left(u_{0}\right)} A_{v_{0}}\left[\sum_{x \in D(e)} \mathcal{R}_{y, x}\right] \bar{A}_{v_{0}} A_{v_{0} x^{-1} w_{0}}, \quad \text { by (43). }
\end{aligned}
$$


Hence, by (46),

$$
\begin{aligned}
\bar{C}_{v_{0} t^{-1} w_{0}} & =(-1)^{\ell(t)} q^{\ell\left(u_{0}\right) / 2} \sum_{\substack{x \in D(e) \\
y \in D(e)}} A_{v_{0}} \overline{\mathcal{P}}_{t, y} \mathcal{R}_{y, x} \bar{A}_{v_{0}} A_{v_{0} x^{-1} w_{0}} \\
& =(-1)^{\ell(t)} q^{\ell\left(u_{0}\right) / 2} \sum_{x \in D(e)} \bar{A}_{v_{0}}\left[\sum_{y \in D(e)} \overline{\mathcal{P}}_{t, y} \mathcal{R}_{y, x}\right] A_{v_{0}} \cdot A_{v_{0} x^{-1} w_{0}},
\end{aligned}
$$

by (35). Since $C_{v_{0} t^{-1} w_{0}}=\bar{C}_{v_{0} t^{-1} w_{0}}$, we see by (45) that (44) is valid. This completes the proof.

Using (3), (5), (38), we have

Corollary 4.6. Let $\sigma \in W e W$. Then there exist $P_{\theta, \sigma} \in \mathbb{Z}[q], \theta \in W e W$, such that in $\mathcal{H}(e)$

(i) $C_{\sigma}=(-1)^{\ell(\sigma)} q^{(\ell(\sigma)-\ell(e)) / 2} \sum_{\theta \in W e W}(-1)^{\ell(\theta)} \bar{P}_{\theta, \sigma} A_{\theta}$,

(ii) $P_{\theta, \sigma}=0$ if $0 \not \leq \sigma$,

(iii) $P_{\sigma, \sigma}=1$.

Problem 4.7. Determine $P_{\theta, \sigma}$ explicitly for $\theta, \sigma \in W e W$. Is $P_{\theta, \sigma} \neq 0$ for $\theta \leq \sigma$ ?

Remark 4.8. Renner [26] has posed the problem of finding the correct polynomials $P_{\theta, \sigma}$ for $\theta, \sigma \in R$. Thus Corollary 4.6 provides a partial solution.

In the special case $W(e)=W_{e}$ the polynomials $P_{\theta, \sigma}$ are related to the relative Kazhdan-Lusztig polynomials $\widetilde{P}_{x, y}^{I}$ studied by Deodhar [6], [8] and Douglass [9].

Corollary 4.9. Let $W(e)=W_{e}=W_{I}, I \subseteq S$, and let $\sigma=$ set $^{-1}, \theta=x e y^{-1} \in$ $W e W$ in standard form. Then $s, t, x, y \in D(e)$ and $P_{\theta, \sigma}=\widetilde{P}_{x, s}^{I} \widetilde{P}_{w_{0} y v_{0}, w_{0} t v_{0}}^{I}$.

Combining Theorems 3.3, 4.1 and 4.5, we have

Theorem 4.10. For $\sigma \in W e W$, let $\bar{A}_{\sigma}, C_{\sigma} \in \mathcal{H}(R)$ be given by (27), (40) respectively. Then:

(i) The map $\tilde{e} A_{\sigma} \rightarrow \tilde{e} \bar{A}_{\sigma}$ yields an involution on $\mathcal{H}(R)$ extending the involution on $\mathcal{H}(W)$.

(ii) If $\widetilde{C}_{\sigma}=\tilde{e} C_{\sigma}$, then $\widetilde{C}_{\sigma}$ is invariant under the involution in (i), and $\widetilde{C}_{\sigma}(\sigma \in R)$ forms a $\mathbb{Z}\left[q^{1 / 2}, q^{-1 / 2}\right]$-basis of $\mathcal{H}(R)$.

\section{REFERENCES}

1. R. W. Carter, Finite Groups of Lie Type: Conjugacy Classes and Complex Characters, Wiley, 1985. MR 87d:20060

2. A. H. Clifford and G. B. Preston, The algebraic theory of semigroups, Vol. 1, Math. Surveys No. 7, Amer. Math. Soc., 1961. MR 24:A2627

3. C. W. Curtis, Representations of Hecke algebras, Astérisque 168 (1988), 13-60. MR 90m:20047

4. C. W. Curtis and I. Reiner, Methods of Representation Theory, Vol. 2, Wiley, 1987. MR 88f:20002

5. V. V. Deodhar, On some geometric aspects of Bruhat orderings. I: A finer decomposition of Bruhat cells, Invent. Math. 79 (1985), 499-511. MR 86f:20045

6. V. V. Deodhar, On some geometric aspects of Bruhat orderings. II: The parabolic analogue of Kazhdan-Lusztig polynomials, J. Algebra 111 (1987), 483-506. MR 89a:20054

7. V. V. Deodhar, A splitting criterion for the Bruhat orderings of Coxeter groups, Comm. Algebra 15 (1987), 1889-1894. MR 88i:20052 
8. V. V. Deodhar, Duality in parabolic set up for questions in Kazhdan-Lusztig theory, J. Algebra 142 (1991), 201-209. MR 92j:20049

9. J. M. Douglass, An inversion formula for relative Kazhdan-Lusztig polynomials, Comm. Algebra 18 (1990), 371-387. MR 91c:20064

10. J. E. Humphreys, Reflection Groups and Coxeter Groups, Cambridge University Press, 1990. MR 92h:20002

11. N. Iwahori, On the structure of a Hecke ring of a Chevalley group over a finite field, J. Fac. Sci. Univ. Tokyo Sect. I 10 (1964), 215-236. MR 29:2307

12. D. Kazhdan and G. Lusztig, Representations of Coxeter groups and Hecke algebras, Invent. Math. 53 (1979), 165-184. MR 81j:20066

13. G. Lusztig, On a theorem of Benson and Curtis, J. Algebra 71 (1981), 490-498. MR 83a:20053

14. J. Okniński and M. S. Putcha, Complex representations of matrix semigroups, Trans. Amer. Math. Soc. 323 (1991), 563-581. MR 91e:20047

15. E. A. Pennell, M. S. Putcha, and L. E. Renner, Analogue of the Bruhat-Chevalley order for reductive monoids, J. Algebra, to appear.

16. M. S. Putcha, A semigroup approach to linear algebraic groups, J. Algebra 80 (1983), 164-185. MR 84j:20045

17. M. S. Putcha, Linear Algebraic Monoids, London Math. Soc. Lecture Note Series., vol. 133, Cambridge Univ. Press, 1988. MR 90a:20003

18. M. S. Putcha, Monoids on groups with BN-pairs, J. Algebra 120 (1989), 139-169. MR 89k:20091

19. M. S. Putcha, Sandwich matrices, Solomon algebras and Kazhdan-Lusztig polynomials, Trans. Amer. Math. Soc. 340 (1993), 415-428. MR 94a:20112

20. M. S. Putcha, Classification of monoids of Lie type, J. Algebra 163 (1994), 636-662. MR 95b:20089

21. M. S. Putcha and L. E. Renner, The system of idempotents and the lattice of $\mathcal{J}$-classes of reductive algebraic monoids, J. Algebra 116 (1988), 385-399. MR 89k:20098

22. M. S. Putcha and L. E. Renner, The canonical compactification of a finite group of Lie type, Trans. Amer. Math. Soc. 337 (1993), 305-319. MR 93g:20123

23. M. S. Putcha and L. E. Renner, Morphisms and duality of monoids of Lie type, J. Algebra 184 (1996), 1025-1040. CMP 1997:1

24. L. E. Renner, Analogue of the Bruhat decomposition for algebraic monoids, J. Algebra 101 (1986), 303-338. MR 87f:20066

25. L. E. Renner, Analogue of the Bruhat decomposition for algebraic monoids. II: The length function and the trichotomy, J. Algebra 175 (1995), 697-714. MR 96d:20049

26. L. E. Renner, Private communication.

27. L. Solomon, The Bruhat decomposition, Tits system and Iwahori ring for the monoid of matrices over a finite field, Geom. Dedicata 36 (1990), 15-49. MR 92e:20035

28. L. Solomon, Reductive monoids, Notes of a talk given at MSRI Workshop on Representations of Reductive Groups over Finite Fields, Berkeley, November 1990.

29. L. Solomon, Algebraic monoids, Notes of a talk given at the Canadian Math. Soc. Meeting, Victoria, December 1991.

30. J. Tits, Buildings of Spherical Type and Finite BN-pairs, Lecture Notes in Math., vol. 384, Springer-Verlag, 1974. MR 57:9866

Department of Mathematics, North Carolina State University, Raleigh, North CarOLINA 27695-8205

E-mail address: putcha@math.ncsu.edu 